\title{
Study of Interaction Between Angiotensin-converting Enzyme ACE and Diuretic Inhibitor by Molecular Modeling
}

\author{
Mesli Fouzia ${ }^{1,2, *}$, Missoum Noureddine ${ }^{2,3}$, Ghalem Said ${ }^{1,2}$ \\ ${ }^{1}$ Department of Chemistry, Faculty of Sciences, Aboubekr Belkaid University, Tlemcen, Algeria \\ ${ }^{2}$ Laboratory of Naturals Products and Bioactives, Tlemcen, Algeria \\ ${ }^{3}$ Department of Science and Technology, Faculty of Technology, Hassiba Benbouali University, Chlef, Algeria
}

Email address:

meslifouzia2018@gmail.com (M. Fouzia)

${ }^{*}$ Corresponding author

To cite this article:

Mesli Fouzia, Missoum Noureddine, Ghalem Said. Study of Interaction Between Angiotensin-converting Enzyme ACE and Diuretic Inhibitor by Molecular Modeling. American Journal of Chemical Engineering. Vol. 7, No. 4, 2019, pp. 113-119. doi: 10.11648/j.ajche.20190704.13

Received: September 18, 2019; Accepted: September 28, 2019; Published: October 28, 2019

\begin{abstract}
Background: Molecular modeling methods are now used routinely to investigate the structure, dynamics, surface properties, and thermodynamics of inorganic, biological, and polymeric systems. Most current drugs target enzymes. This theoretical approach enables to predict the mode of interaction of a ligand with its receptor. The inhibition of Angiotensinconverting enzyme (ACE) is an important approach in the treatment of Heart failure (HF). The three families of Diuretic are used for inhibiting ACE. Our work is the study of molecular interaction between the enzyme (Angiotensin Converting) and the substrates (inihibitor for ACE). Various tools of molecular modeling are used to carry out this work (molecular mechanics, molecular dynamics and molecular docking) (MOE). The introduction of bulky groups causes a conformational rearrangement in thea ctive site pocket, which will probably be reinforced and thus complement its activity. The results obtained from this work, in which the inhibitions of Angiotensin Converting by molecular modeling methods have been demonstrated. In conclusion, taking into account the results obtained in this study, inhibition of Angiotensin Converting by molecular modeling methods has been elucidated, which allow us to conclude that Bumetanide (loopDiuretic) when water is included in the docking simulation has a better inhibition of Angiotensin Converting.
\end{abstract}

Keywords: Molecular Modelling, Angiotensin, Converting Enzyme (ACE), Diuretic Inhibitor, MOE (Molecular Operating Environment)

\section{Introduction}

Heart failure (HF), often referred to as congestive heart failure (CHF), occurs when the heart is unable to pump sufficiently to maintain blood flow to meet the body's needs. [1] Signs and symptoms commonly include shortness of breath, excessive tiredness, and leg swelling. The shortness of breath is usually worse with exercise, while lying down, and may wake the person at night. A limite dability to exercise is also a common feature. Chest pain, including angina, does not typically occur due to Heart Failure. [2-3] We quote the works of investigating Heart Failure Disease by Studying Interaction between Angiotensin-Converting Enzyme
(ACE) and Different Inhibitors of captopril and beta bloker by Mesli et al. [18-19] In best of our knowledge no studies interaction by docking between these inhibitor (Diuretic) and ACE enzyme has been done. The purpose of this study is to minimize the formation of the complex and consequently to delay its progression. In order to rationalize the properties of the inhibitors and to determine the reaction processes complementarity (better activity). These involving this compound. Our objective main thing in this research and to determine the mode of interaction of the complex for the binding of the inhibitor to the enzyme, with better results will probably help with the development of an effective therapeutic tool to fight against the development of the disease Cardiac Failure. 


\section{Materials and Methods}

\subsection{Angiotensin-Converting ACE Enzyme}

Angiotensin-converting enzyme, or ACE, is a central component of the renin-angiotensin system (RAS), which controls blood pressure by regulating the volume of fluids in the body. [4-5] It converts the hormone angiotensin I to the active vasoconstrictor angiotensinII. Therefore, ACE indirectly increases blood pressure by causing blood vessels to constrict. Other less known functions of ACE are degradation of bradykinin. [6] and amyloid beta-protein. ACE is a zinc metalloenzyme. The zinc ion is essential to its activity, since it directly participates in the catalysis of the peptide hydrolysis.

\subsection{Angiotensin-Converting ACE Inihibitors}

Anangiotensin-converting-enzyme inhibitor (ACE inhibitor) is a pharmaceutical drug used primarily for the treatment of hypertension (elevated blood pressure) and congestive heart failure. This group of drugs causes relaxation of blood vessels as well as a decrease in blood volume, which leads to lower blood pressure and component of the renin-angiotensin system. ACE inhibitors also have beneficial effects.

On left ventricular hypertrophy, another clinical marker of therapy in the hypertensive patient.

ACE inhibitors also avoid some of the detrimental metabolic effects of other antihypertensive medications, such as dyslipidemia, glucose intolerance, and hyperinsulinemia. Finally, ACE inhibitors may be used in African Americans successfully, although higher doses or combination with a diuretic may be necessary for optimal effect. The inhibitors for ACE chosen are given in table 1 .

Table 1. Physico-Chemical properties of Diuretic for ACE.

\begin{tabular}{|c|c|c|c|c|c|}
\hline n & Name & IUPACname & PubChemCID & Molarmass & Formula \\
\hline 1 & Furosemide & 4-chloro-2-(furan-2-ylmethylamino)-5-sulfamoylbenzoicacid & 3440 & 330.739 & $\mathrm{C}_{12} \mathrm{H}_{11} \mathrm{ClN}_{2} \mathrm{O}_{5} \mathrm{~S}$ \\
\hline 2 & Bumetanide & 3-butylamino-4-phenoxy-5-sulfamoyl-benzoicacid & 2471 & 364.416 & $\mathrm{C}_{17} \mathrm{H}_{20} \mathrm{~N}_{2} \mathrm{O}_{5} \mathrm{~S}$ \\
\hline 3 & Piretanide & 4-phenoxy-3-pyrrolidin-1-yl-5-sulfamoylbenzoicacid & 4849 & 362.40 & $\mathrm{C}_{17} \mathrm{H}_{18} \mathrm{~N}_{2} \mathrm{O}_{5} \mathrm{~S}$ \\
\hline 4 & Torasemide & 1-[4-(3-methylanilino)pyridin-3-yl]sulfonyl-3-propan-2-ylurea & 41781 & 348.421 & $\mathrm{C}_{16} \mathrm{H}_{20} \mathrm{~N}_{4} \mathrm{O}_{3} \mathrm{~S}$ \\
\hline 5 & Hydrochlorothiazide & 6-chloro-1,1-dioxo-3,4-dihydro-2 $H$-1,2,4-benzothiadiazine-7-sulfonamide & 3639 & 297.728 & $\mathrm{C}_{7} \mathrm{H}_{8} \mathrm{ClN}_{3} \mathrm{O}_{4} \mathrm{~S}_{2}$ \\
\hline 6 & Bendroflumethiazide & $\begin{array}{l}\text { 3-Benzyl-1,1-dioxo-6-(trifluoromethyl)-3,4-dihydro- } 2 H \text {-1,2,4- } \\
\text { benzothiadiazine-7-sulfonamide }\end{array}$ & 2315 & 421.409 & $\mathrm{C}_{15} \mathrm{H}_{14} \mathrm{~F}_{3} \mathrm{~N}_{3} \mathrm{O}_{4} \mathrm{~S}_{2}$ \\
\hline 8 & Chlortalidon & 2-chloro-5-(1-hydroxy-3-oxo-2H-isoindol-1-yl)benzenesulfonamide & 2732 & 338.762 & $\mathrm{C}_{14} \mathrm{H}_{11} \mathrm{ClN}_{2} \mathrm{O}_{4} \mathrm{~S}$ \\
\hline 9 & Indapamide & 4-chloro-N-(2-methyl-2,3-dihydroindol-1-yl)-3-sulfamoyl-benzamide & 3702 & 365.835 & $\mathrm{C}_{16} \mathrm{H}_{16} \mathrm{ClN}_{3} \mathrm{O}_{3} \mathrm{~S}$ \\
\hline
\end{tabular}

\subsection{Preparation and Optimization of Both Enzyme and Inhibitors}

Download of ACE was done from data base Bookhaven Protein (www.rcsb.org/pdb) (code4BZR). With threedimensional structure obtained byX-ray diffraction

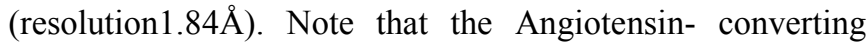
enzyme (ACE) crystallizes as a monomer (Figure 1) with 589 residues and 4726 atoms. Compound s of inhibitors for ACE were downloading from Pub Chem data base. (www.pubchem.com) see table 2 Using MOE software (Molecular operating environment). [7] We select the active site in the enzyme and we minimize the energy of both enzyme and molecules. Energy minimizing was done under following conditions: Temperature $=300^{\circ} \mathrm{K}, \mathrm{pH}=7$, the geometry was performed using the field strengths in the MMFF94x implanted in MOE. [7] and Hamiltonian AM1. Figure 2 shows the active site of the enzyme with molecule of co-crystallization Minimized energy of ligands and their toxicity are obtained by MOE software (table 3 ).

Table 2. Diuretic inhibitors used for ACE.

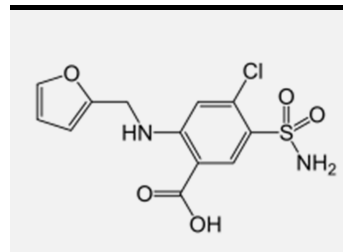

B-blocker1(CID3440)

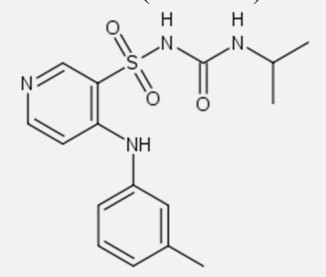

B-blocker4(CID41781)

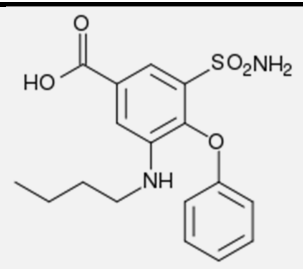

B-blocker2(CID2471)<smiles>NS(=O)(=O)c1cc2c(cc1Cl)NCNS2(=O)=O</smiles>

B-blocker5(CID3639)

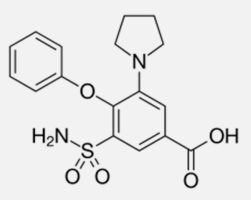

B-blocker3(CID4849)<smiles>NS(=O)(=O)c1cc2c(cc1C(F)(F)F)NC(Cc1ccccc1)NS2(=O)=O</smiles>

B-blocker6(CID2315) 


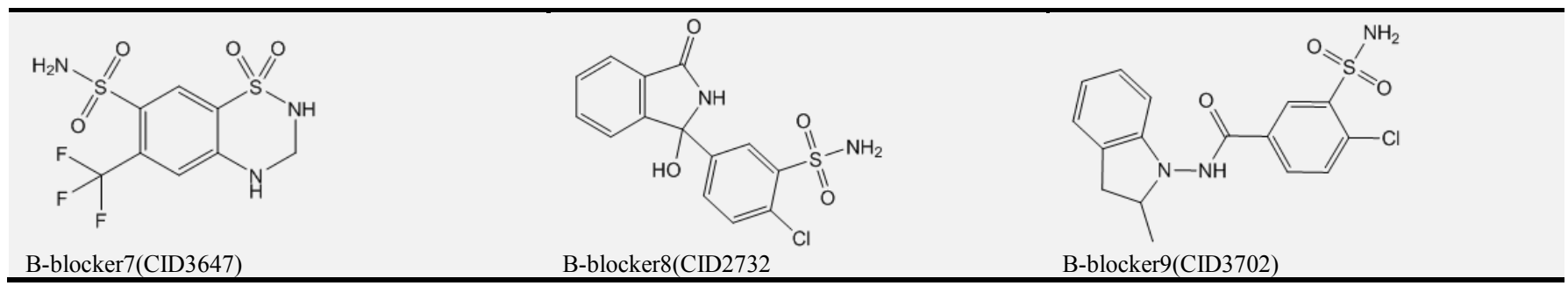

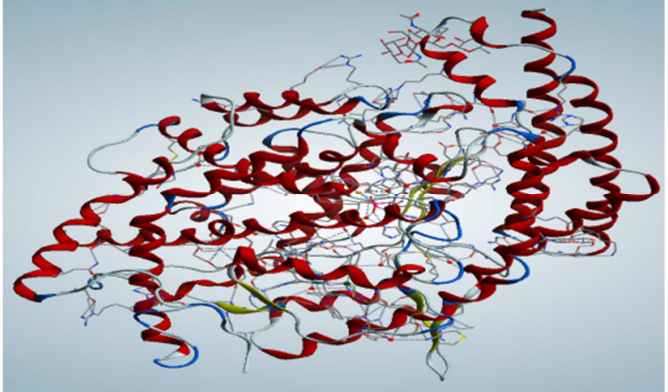

Figure 1. Simplified model of (ACE).

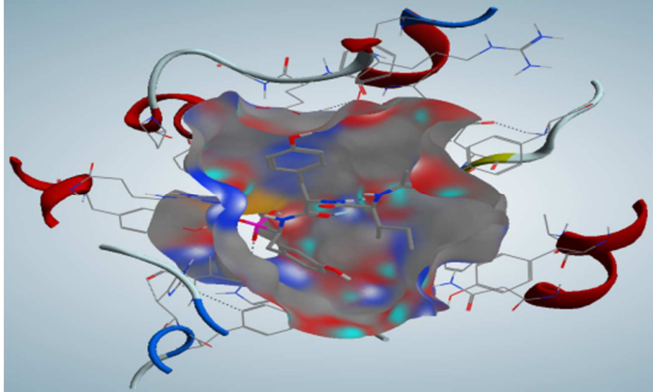

Figure 2. Site active enzyme isolated.

Table 3. Minimization energy of molecules (Kcal/mol)A/; Diuretic.

\begin{tabular}{|c|c|c|c|c|c|}
\hline A/Ligand & Molecules & Energies (Kcal/mol) & $\log P$ & LogS & Toxcicity \\
\hline 1 & Furosemide & $4.88308 \mathrm{e}+001$ & 1.92 & -3.92 & No \\
\hline 2 & Bumetanide & $8.40241 \mathrm{e}+001$ & 2.80 & -4.49 & No \\
\hline 3 & Piretanide & $9.78951 \mathrm{e}+001$ & 2.18 & -4.13 & No \\
\hline 4 & Torasemide & $-2.89409 \mathrm{e}+001$ & 2.53 & -3.14 & No \\
\hline 5 & Hydrochlorothiazide & $-2.03037 \mathrm{e}+001$ & 0.74 & -1.86 & No \\
\hline 6 & Bendrofluméthiazide & $1.20101 \mathrm{e}+001$ & 3.03 & -4.19 & No \\
\hline 7 & Hydrofluméthiazide & $-1.61202 \mathrm{e}+001$ & 1.42 & -2.18 & No \\
\hline 9 & Indapamide & $8.73069 \mathrm{e}+001$ & 3.18 & -4.62 & No \\
\hline
\end{tabular}

These ligands are able to present a very important biological activity in accordance with the rule of Lipinski., et al.(1997). [8]

\subsection{Docking and Building Complexes}

The next step, after the construction of the ligand, is the positioning of this molecule in the active site of Angiotensin Converting (ACE). For this, we used the Molecular Docking Module using MOE software. [7] Once the ligand-receptor complex is formed, it will adapt the most stable conformation, i.e. the lowest energy level. The purpose of the Dock application is looking at favorable conformational binding between medium size ligands and a not so soft macromolecular target, which is usually a protein. [9] For each ligand, a number of conformations called poses were generated to identify favorable binding modes. The search for binding modes is generally constrained to a small specific region of the receptor called the active site.

\section{Results and Discussion}

Table 4. Energy Balance of 9 complexes Diuretic-ACE Without Water (Kcal/mol).

\begin{tabular}{|c|c|c|c|c|c|c|c|}
\hline Mol & score & Rmsd-refine & E-Conf & E-PLACE & E-SCORE1 & E-REFINE & E-SCORE2 \\
\hline Ligref & -13.6675 & 2.7837 & -222.2882 & -107.0895 & -18.8538 & -76.8066 & -13.6675 \\
\hline Complexe-1 & -7.6868 & 2.1833 & -88.1296 & -81.4621 & -15.8095 & -35.2535 & -7.6868 \\
\hline Complexe-2 & -7.6617 & 1.8771 & -68.6812 & -92.1191 & -17.5884 & -46.08067 & -7.6617 \\
\hline Complexe-3 & -7.3746 & 2.3918 & -55.9997 & -87.6524 & -15.7990 & -40.6328 & -7.3746 \\
\hline Complexe-4 & -6.3973 & 2.9382 & -204.0534 & -66.4851 & -10.3818 & -20.3048 & -6.3973 \\
\hline Complexe-5 & -4.0564 & 3.9171 & -198.1333 & -83.6652 & -11.7637 & -11.9169 & -4.0564 \\
\hline Complexe- 6 & -5.6952 & 2.0831 & -152.9348 & -113.2216 & -12.4956 & -25.0720 & -5.6952 \\
\hline Complexe-8 & -5.1020 & 2.4142 & -73.0422 & -82.4938 & -11.2163 & -22.6544 & -5.1020 \\
\hline Complexe-9 & -6.9100 & 2.8700 & 28.5760 & -127.3632 & -11.2711 & -23.4986 & -6.9100 \\
\hline
\end{tabular}

$\mathrm{S}$ : the final score; is the score of the last step, rmsd refine: the mean square deviation between the laying before refinement and after refinement pose, E conf: energy conformer, E_place: score of the placement phase, E_scor1: score the first step of notation, E_refine: score refinement step and number of conformations generated by ligand E_scor2: score the first step notation, number of poses: Number of conformations. [10] 
Table 5. EnergyBalanceof9 complexesDiuretic-ACEinWater (Kcal/mol).

\begin{tabular}{llllllll}
\hline Mol & score & Rmsd-refine & E-Conf & E-PLACE & E-SCORE1 & E-REFINE & E-SCORE2 \\
\hline Ligref & -13.6675 & 2.7837 & -222.2882 & -107.0895 & -18.8538 & -76.8066 & -13.6675 \\
Complexe-1 & -8.5379734 & 1.50055301 & --88.48154 & -92.7198715 & -16.066850 & -31.362251 & -8.5379734 \\
Complexe-2 & -8.79894733 & 2.52560592 & -68.328186 & -80.024437 & -18.635086 & -38.890029 & -8.79894733 \\
Complexe-3 & -8.45710182 & 1.44391918 & -56.209110 & -101.528679 & -20.234661 & -40.956218 & -8.45710182 \\
Complexe-4 & -7.67036247 & 2.15212536 & -214.07959 & -91.324501 & -13.784414 & -15.639841 & -7.67036247 \\
Complexe-5 & -5.82894754 & 2.29741526 & -197.84294 & -134.48494 & -16.028841 & -8.7950677 & -5.82894754 \\
Complexe-6 & -6.58859253 & 2.38762617 & -149.99552 & -105.247528 & -19.002553 & -9.3228254 & -6.58859253 \\
Complexe-7 & -6.54932451 & 4.34839535 & -198.21467 & -90.1178513 & -19.025461 & -21.010179 & -6.54932451 \\
Complexe-8 & -6.67853212 & 2.72179699 & -70.063423 & -88.8947983 & -15.839345 & -18.342468 & -6.67853212 \\
Complexe-9 & -6.54761505 & 2.44884992 & 34.4425278 & -66.3137589 & -16.879350 & -7.0040726 & -6.54761505 \\
\hline
\end{tabular}

The results given in tables 4 and 5 show that the orientation of the ligands play significant sasignificant role for the positioning of the ligands in the actives site ite of the enzyme, one can conclude that the introduction of bulky groups causes arearrangement of conformation in side the cavity of the active site, which will be probably the complementarity and consequently the activity. [11] 2D molecular method of the screen has been attributed to the MOE (Molecular Operating Environment) software, which is designed to visualize the active sites of the complex (proteinligand). The ligand is prepared and made with an improved 2D depiction layout algorithm, and protein residues version are arranged around it to indicate links spatial proximity. [12]
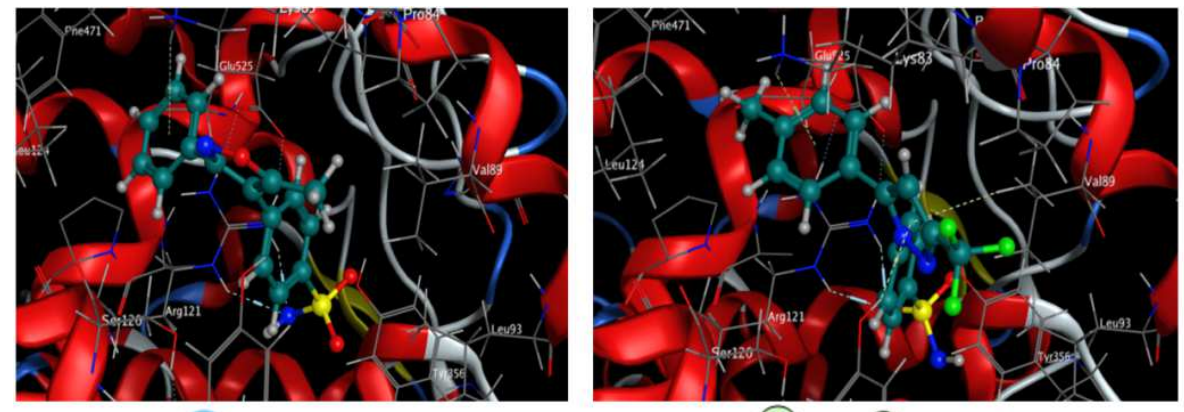

(79)

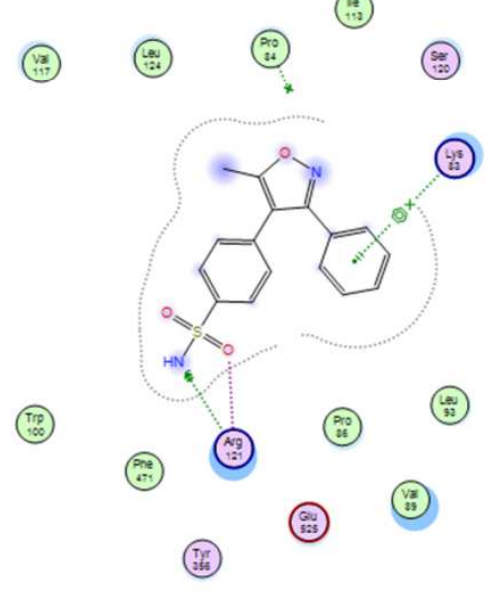

(7ix)

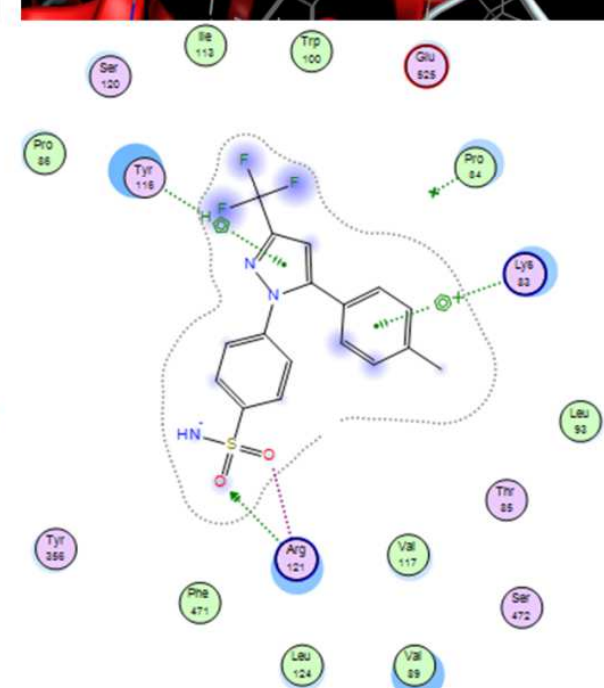

Residues are marked with their amino acid code of 3 letters, and job classification classificatin. [13-14] If there are multiple channels in the system, the positions are prefixed by the letters of the alphabet. Interactions between $2.5 \AA$ and 3.1 $\AA$ are considered high and those between $3.1 \AA$ and $3.55 \AA$ are average. $3.55 \AA$ interactions are weak. [15]

\subsection{Synthetic Inhibitors Approach Without Water}

These results show that the complex-1has the lowest energy $(-7.6868 \mathrm{Kcal} / \mathrm{mol})$ and is more active than complex$2(-7.6617 \mathrm{Kcal} / \mathrm{mol})$, which is more active than complex-3($7.3746 \mathrm{Kcal} / \mathrm{mole})$.

a. Diagram interaction of complex-1 (ACE + Furosemide).

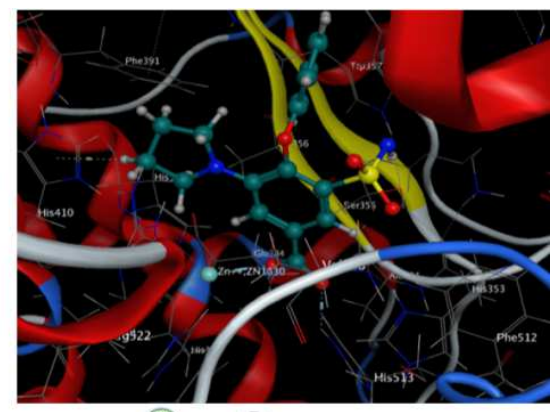

(iiii)

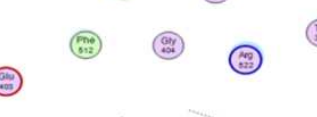

(99)

(10).

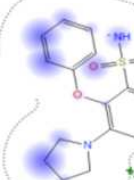

(10)

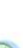

(2)
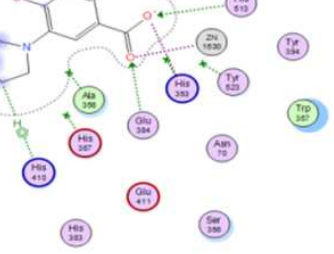

b. Diagram interaction of complex-2 (ACE + Bumetanide). c. Diagram interaction of complex-3 (ACE + Piretanide).

Figure 3. Diagrams of interactions (enzyme-ligands) 3 complex ( $a, b, c)$.

For complex 1: Furosemide interacts with the amino acid [TYR 523 (A) H-acceptor, GLU 384 (A) H-acceptor; ALA 356 (A) pi-H] at a distance of $2.83,2.97,4.47 \AA$ respectively (for the 1st, 2nd strong interaction, and 3th weak interaction), with the exixtence of five electric forces ASP 358, HIS 353, HIS 513, HIS 387 and HIS 410and interaction with Zink Zn 
1630 (metal, ionic) at a distance of 2.62, 2.47 for metal and ionic strong interaction for the1st wich suggesting that Furosemide can inhibit Angiotensin - Converting (ACE) and interfere with [TYR 523 (A) H-acceptor, GLU 384 (A) Hacceptor; ALA 356 (A) pi-H]. [16]

For complex 2: Bumetanide has an interaction with the amino acids [HIS 513 (A) H-acceptor, GLU 384 (A) Hacceptor, HIS 353 (A) ionic, ALA 356 (A) pi-H] at a distance of $2.94,2.84,3.33,4.62 \AA$ respectively (for the 1st, 2nd strong interaction, 3th average interaction and 4th weak interaction), with the exixtence of five electric forces HIS 410, HIS 387, TYR523, HIS 353, ASP 358 and interaction with Zink Zn1630 ionic at a distance of $2.30 \AA$ for metal and ionic which suggests that Bumetanide can inhibit Angiotensin - Converting (ACE) and interfere with acid [HIS 513 (A) H-acceptor, GLU 384 (A) H-acceptor, HIS 353 (A) ionic, ALA 356 (A) pi-H] [16].

For complex 3: Piretanide interacts with amino acids [GLU 384 (A) H-acceptor, HIS 513 (A) H-acceptor, HIS 353 (A), HIS 410 (A) H-pi] at a distance of 2.85.3,14, 3.57; 4.09 $\AA$ respectively (for the $1 \mathrm{st}, 2 \mathrm{nd}$ strong interaction, 3nd average interaction, and 4 th weak interaction), with the exixtence of five electric forces

HIS 387, HIS 353; TYR523, ASP358, ALA356 and interaction with Zink Zn 1630 (metal, ionic) at a distance of $2.32 \AA$ for metal and ionic, which suggesting that Piretanide can inhibit Angiotensin - Converting (ACE) and interfere with [GLU 384 (A) H-acceptor, HIS 513 (A) H-acceptor, HIS 353 (A), HIS 410 (A) H-pi]. [16]

\subsection{Synthetic Inhibitors Approach in Water}

These results show that the complex-2 has the lowest energy $(-8.79894733 \mathrm{Kcal} / \mathrm{mol})$ and is more active than
complex-1(-8.5379734Kcal $/ \mathrm{mol})$, which is more active than complex-3 (-8.45710182Kcal $/ \mathrm{mole})$.

For complex2: Bumetanide interacts with the amino acid [GLU384(A)H-acceptor; HIS513(A)H-acceptor; ARG522[(A)H-acceptor; ionic)]; HIS353(A) ionic] at a distance of 2.96,3.06,3.19;3.65,3.19 respectively (for the $1 \mathrm{st}, 2 \mathrm{nd}, 3 \mathrm{rd}, 5^{\text {th }}$ strong interaction and $4^{\text {th }}$ weak interaction) and interaction with Zink Zn1630 (metal, ionic) at a distance of 2.38 for metal and ionic strong interaction wich suggesting that Bumetanide can inhibit Angiotensin-Converting (ACE) and interfere with [GLU384(A)H-acceptor; HIS513(A)Hacceptor; ARG522[(A)H-acceptor; ionic)]; HIS353(A)ionic]. [16]

For complex1: Furosemide interacts with the amino acid [TYR523(A) H-acceptor; GLU384(A)H-acceptor; HIS410(A)pi-pi] at a distance of $2.93 ; 3.23 ; 3.78 \AA$ respectively (for the1st strong interaction, $2^{\text {nd }}$ average interaction, and 3th weak interaction) and interaction with Zink Zn1630 (metal, ionic) at a distance of 2.65,2.59for metal and ionic strong interaction wich suggesting that Furosemide can inhibit Angiotensin-Converting (ACE) and interfere with [TYR523(A)H-acceptor; GLU384(A) H-acceptor; HIS410(A)pi-pi]. [16]

For complex3: Piretanide interacts with the amino acid [GLU384(A)H-acceptor; HIS513(A)H-acceptor; HIS353 (A)ionic; HIS410(A)H-pi] at a distance of $2.91,3.17,3.68,4.07 \AA$ respectively (for the $1 \mathrm{st}, 2^{\text {nd }}$ strong interaction, and 3thand4th weak interaction) and interaction with Zink Zn1630 (metal, ionic) at a distance of 2.33 for metal and ionic strong interaction wich suggesting that Piretanide can inhibit Angiotensin-Converting (ACE) and interfere with [GLU384(A)H-acceptor; HIS513(A)Hacceptor; HIS353 (A)ionic; HIS410(A)H-pi]. [16]

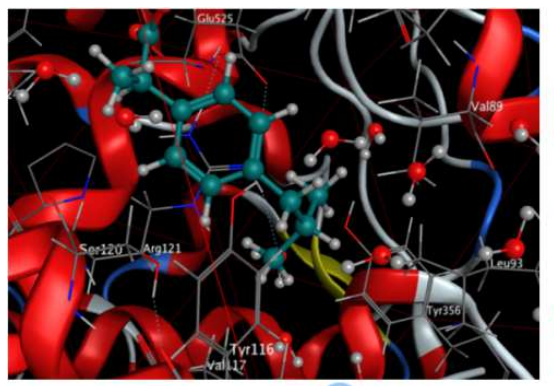

(3)

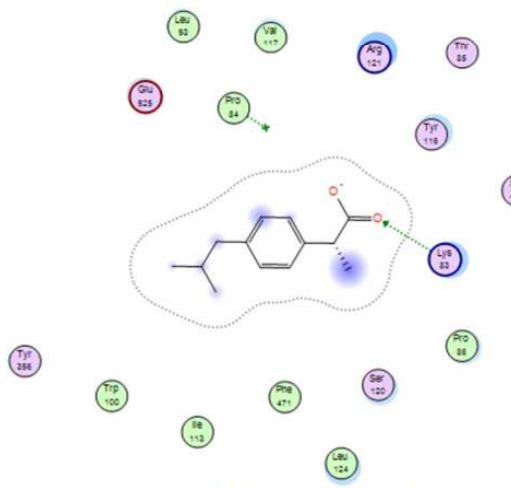

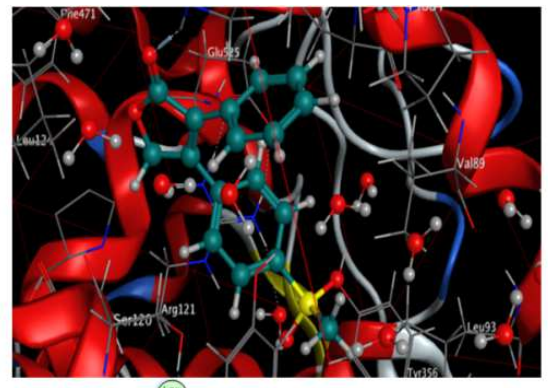

(28) (अ) (ii) (ii)

(:)

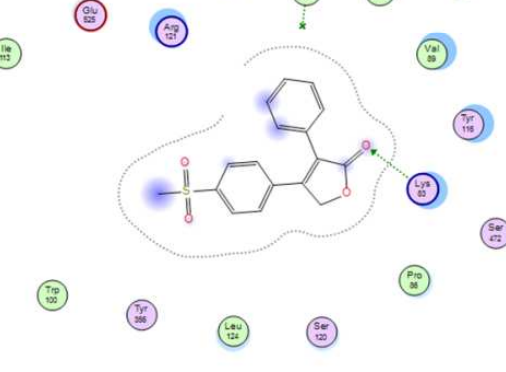

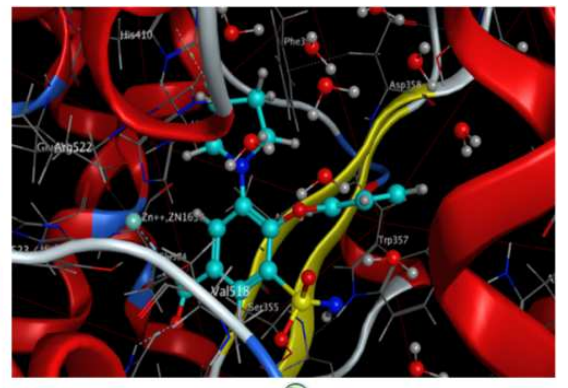

(3)

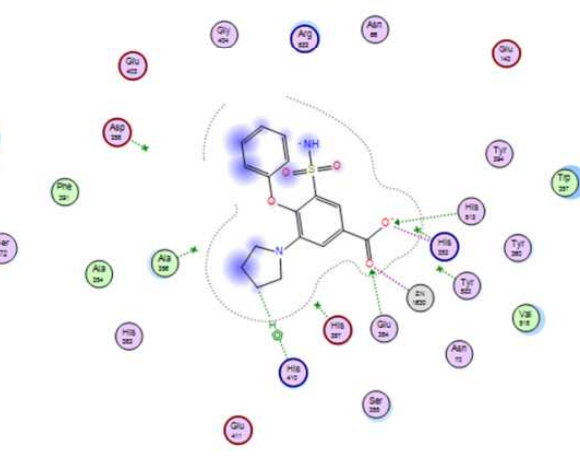

a. Diagram interaction of complex-2 (ACE +Bumetanide). b. Diagram interaction of complex-1 (ACE + Furosemide). c. Diagram interaction of complex-3 (ACE + Piretanide).

Figure 4. Diagram of interactions (enzyme-ligands) 3 complex $(4 a, 4 b, 4 c)$. 
The value of IC50 with the inhibitor of the K27cocrystallizationis $14.4 \mathrm{nM}$. [17].

Energy (Furosemide- $7.6868 \mathrm{Kcal} / \mathrm{mol}<$ Bumetanide7.661 $\mathrm{Kcal} / \mathrm{mol}<$ Piretanide-7.3746Kcal $/ \mathrm{mol}$ ).

Energy (Bumetanide-8.79894733Kcal $/ \mathrm{mol}<$ Furosemide$8.5379734 \mathrm{Kcal} / \mathrm{mol}<$ Piretanide- $8.45710182 \mathrm{Kcal} / \mathrm{mol}$ ).

Bumetanide (Diuretic) when water is included in the docking simulation will be the best inhibitors to slow the evolution of the pathology studied (heart failureHF).

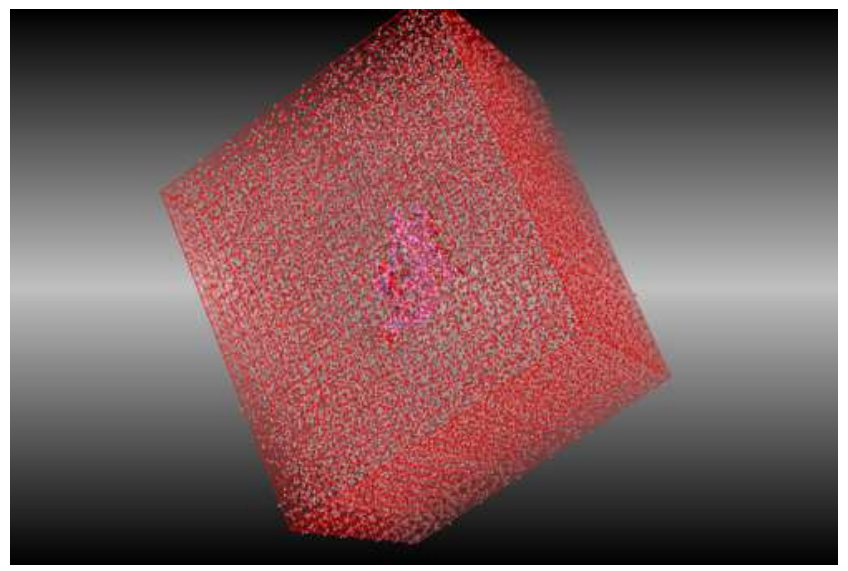

Figure 5. Solvation Ligand-Substrat in Cube box.

Whichever molecule a protein binding complex could be found, it is inherently emotional to be rigidly replaced by a body bond. As a result, the prediction of mediation displacement requires an exact equilibrium. Refinements of certain terms of the force field describing the water molecule are imperative to ensure good results. For example, the explicit treatment of the electronic polarizability of water molecules can be included as an additional term in an empirical force field. The presence of water is sometimes paramount to ensure a relay between the ligand and the active site.

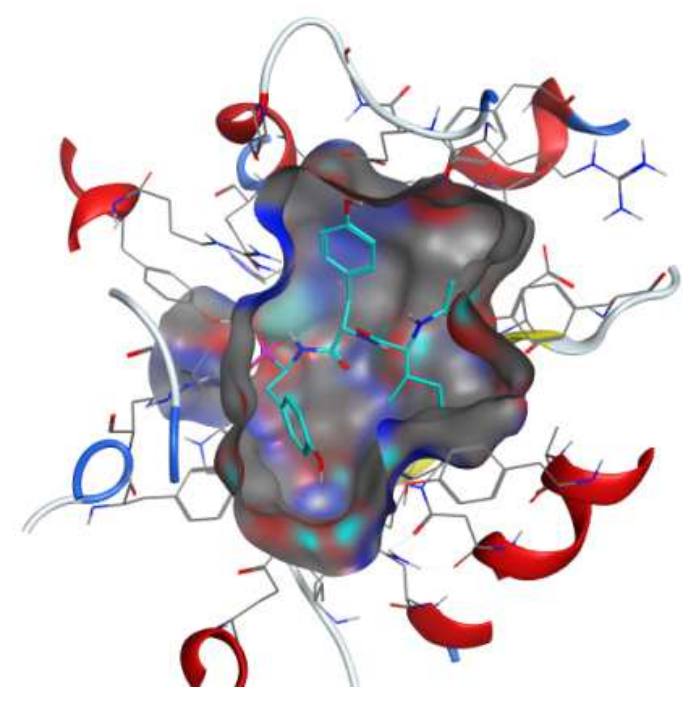

Figure 6. Size of the enzyme cavity.

Note that we can discuss complementarity increasing or decreasing in the range of dimensions of the active site pocket, in our case with a deep geometry $19.06 \AA$ an opening and $15.92 \AA$, this pocket shrunk up a width $10.07 \AA$ (Figure 6). Taking into account different geometrical constraints, the approach considered inhibitors may influence the complementarity and later activity.

The examination of the enzymatic cavity confirms that the structure of Furosemide with the groupings of the atoms $\left(\mathrm{O}_{6}\right.$, $\mathrm{O}_{7}$ ) presents a strong interaction hydrogen bond with [TYR523(A)H-acceptor, GLU384(A)H-acceptor] and for Bumetanide with the atom $\left(\mathrm{O}_{5}, \mathrm{O}_{6}, \mathrm{~N}_{8}\right)$ present a strong interaction hydrogen bond with [GLU384(A)H-acceptor; HIS513(A)H- acceptor; ARG522ionic)]; and one better complementarity with Angiotensin Converting enzyme (ACE).

\section{Conclusion}

The results obtained from this work, into which the inhibition of Angiotensin Converting (ACE) by molecular modeling methods was elucidated, allow us to conclude that inhibitor-2Bumetanide (LoopDiuretic) when water is included in the docking simulation presents a more optimized inhibition of Angiotensin Converting (ACE) for the treatment of Heart Failure (HF). We can conclude that Bumetanide (LoopDiuretic) present better interaction of Angiotensin Converting Enzyme in presence of water molecules while docking and consequently can be the best inhibitor candidate to be in vitro and in vivo investigated.

\section{Acknowledgements}

The authors thank director of Laboratory $\operatorname{Pr}$ Said GHALEM- LASNABIO for his financial support and ensure that there is no conflict of interest regarding this paper. Authors also thank anonymous reviewers for their suggestions.

\section{References}

[1] R. O Bonow., et al. "ACC/AHA clinical performance measures for adults with chronic heart failure," Journal of the Ameri-can College of Cardiology 46.6 (2005), 1144-1178.

[2] S. A Hunt., et al. "Liver function abnormalities, clinical profile, and outcome in acute decompensate heart failure,". European Heart Journal 34.10 (2013), 742-749.

[3] K. Dickstein, et al. "ESC Guide lines for the diagnosis and treatment of acute and chronic heart failure 2008: the Task Force for the Diagnosis and Treatment of Acute and Chronic Heart Failure 2008 of the European Society of Cardiology. Developed in collaboration with the Heart Failure Association of the ESC (HFA) and endorsed by the European Society of Intensive Care Medicine (ESICM)," European Heart Journal 29.19 (2008), 2388-2442.

[4] L. Skeggs., et al. "The preparation and function of the hypertension-converting enzyme," Journal of Experimental Medicine103.3 (1956), 295-299. 
[5] M. AZaman., et al. "Drugs targeting the renin-angiotensin-aldosterone system," Nature Reviews Drug Discovery 1.8 (2002), 621-636.

[6] E. D. Sturrock., et al. "Structure of AngiotensinI- Converting En-zyme," Cellular and Molecular Life Sciences 61.21 (2004), 2677-2686.

[7] Molecular Operating Environment (MOE), 2013.08; Chemical Computing GroupInc., Canada (2013).

[8] J. P. Powers, D. E. Piper, LiY, V. Mayorga, J. Anzola, J. M. Chen, et al. "SARand mode of action of novel non-nucleoside inhibitors of hepatitis CNS5bRNA polymerase," J. Med Chem, 2006, 49 (3).

[9] J. Goto., et al. "ASEDock-Docking Based on Alpha Spheres and Excluded Volumes". Journal of Chemical Information and Modeling48.3 (2008), 583-590.

[10] A. M Manikrao., et al. "Docking Studies of few C3Substituted Azapteridines as Hepatitis CVirus RNADependent RNAPoly-merase inhibitors," Journal of Computational Methods in Mo-lecular Design, 1.4 (2011), 3545.

[11] F. Mesli, S. Ghalem. "Comparative studies of Chromen Derivatives by Using Numerical Methods," Asian Journal of chemistry, 2017, 29 (7), 1405-1412.

[12] P. Labute, C. Williams, M. Feher, E. Sourial, J. M. Schmidt. "Flexible alignment of small molecules," J. Med. Chem, 2001, 1483-1490. Doi: 10.1021/jm0002634.
[13] A. M Clark, P. Labute, M. Santavy. "2D Structure Depiction," J. Chem. 46, Inf. Model, 2006, 1107-1123.

[14] A. MClark., et al."Detectionand Assignment of Common Scaf-foldsin Project Databases of Lead Molecules," Journal of Me-dicinal Chemistry, 52.2 (2008), 469-483.

[15] D. W Ritchie., et al." Protein docking using pherical polar Fourier correlations,” Proteins 39.2 (2000), 178-194.

[16] H. K Yamaguchi., et al. "Structural insight into the ligandreceptor interaction between 6- methylsulfinyl) hexylisothio cyanate and multidrug esistance-associated protein1 nucleotide-binding domain1," International Journal of Computational Bioinformatics and In Silico Modeling, 3 (2014), 310-314.

[17] N. Ioanna., et al. "I dentification of ACE pharmacophore in the phosphonopeptide metabolite K-26," Bioorganic and Medicinal Chemistry Letters, 18.10 (2008), 3068-3071.

[18] F. Mesli, N. Missoum, S. Ghalem. "Study of Interaction between Angiotensin-Converting Enzyme (ACE) and Beta Blocker Inhibitors Including Solvatation Parameter with Molecular Modeling," Acta Scientific Medical Sciences, 2019 July3 (7), 159-166.

[19] F. Mesli, S. Bouchentouf, A. Ghomri, N. Missoum, S. Ghalem. "Investigating Heart Failure Disease by Studying Interaction between Angiotensin Converting Enzyme (ACE) and Different Inhibitors including Solvatation Parameter with Molecular Docking," Acta Scientific Pharmaceutical Sciences, 2018 May2 (5), 22-29. 